\title{
Pulmonary Scintigraphy for the Diagnosis of Acute Pulmonary Embolism: A Survey of Current Practices in Australia, Canada, and France
}

\author{
Pierre-Yves Le Roux ${ }^{1,2}$, Matthieu Pelletier-Galarneau ${ }^{3}$, Romain De Laroche ${ }^{1}$, Michael S. Hofman ${ }^{2}$, Lionel S. Zuckier ${ }^{3}$, \\ Paul Roach ${ }^{4}$, Jean-Philippe Vuillez ${ }^{5}$, Rodney J. Hicks ${ }^{2}$, Grégoire Le Gal ${ }^{3,6}$, and Pierre-Yves Salaun ${ }^{1}$ \\ ${ }^{1}$ Université Européenne de Bretagne, Université de Brest, EA3878 (GETBO) IFR 148, CHRU de Brest, Service de Médecine \\ Nucléaire, Brest, France; ${ }^{2}$ Peter MacCallum Cancer Centre, University of Melbourne, Melbourne, Victoria, Australia; ${ }^{3}$ The Ottawa \\ Hospital, University of Ottawa, Ottawa, Canada; ${ }^{4}$ Royal North Shore Hospital, Sydney, New South Wales, Australia; ${ }^{5}$ CHU de \\ Grenoble, Grenoble, France; and ${ }^{6}$ Université Européenne de Bretagne, Université de Brest, INSERM CIC 05-02 IFR148, CHRU de \\ Brest, Département de Médecine Interne et de Pneumologie, Brest, France
}

There are currently no data published regarding the proportion of nuclear medicine centers using SPECT or SPECT/CT rather than planar ventilation/perfusion (V/Q) imaging in patients with suspected acute pulmonary embolism (PE). Furthermore, the reporting criteria used for interpretation of both planar and SPECT V/Q scans are variable and data are lacking regarding which criteria are commonly used in various centers. The aim of this study was to assess current practices regarding the performance and interpretation of lung scintigraphy across 3 different countries. Methods: A short online survey composed of simple multiple-choice questions was distributed to nuclear medicine departments in Australia, Canada, and France during the period April to December 2014. The survey covered image acquisition, interpretation criteria for SPECT and planar images, and use of pseudoplanar images and radiopharmaceuticals. Information was initially solicited by 2 sets of e-mails, which pointed to the survey internet link. Departments were subsequently contacted by telephone. A single response per department was consolidated. Results: Three hundred thirty-one responses were collected (Australia, 74; Canada, 48; and France, 209). Twenty-eight percent of centers indicated use of $V / Q$ planar imaging alone whereas $72 \%$ of centers included some form of SPECT in their acquisition protocol for evaluation of PE, specifically V/Q SPECT in $36 \%, \mathrm{~V} / \mathrm{Q}$ SPECT/CT in $29 \%$, Q SPECT/CT in $2 \%$, and both V/Q planar and SPECT in $5 \%$, with a strong variability among countries. The most commonly used criteria for SPECT interpretation were the those of the European Association of Nuclear Medicine (60\%). Criteria used for planar interpretation were heterogeneous (European Association of Nuclear Medicine criteria, 35\%; Prospective Investigation of Pulmonary Embolism Diagnosis study, 29\%; no standardized criteria, $21 \%)$. Sixty-three percent of departments used pseudoplanar images in addition to SPECT images. Conclusion: In the 3 countries surveyed, SPECT has largely replaced planar imaging for evaluation of PE, with almost half of the SPECT studies incorporating a CT acquisition. Criteria used for interpretation are inconsistent, especially for planar imaging.

\footnotetext{
Received Apr. 29, 2015; revision accepted Jun. 18, 2015.

For correspondence or reprints contact: Pierre-Yves Le Roux, Service de Médecine Nucléaire, CHRU de Brest, 29609 Brest Cedex, France.

E-mail: pierre-yves.leroux@chu-brest.fr

Published online Jul. 1, 2015.

COPYRIGHT (C) 2015 by the Society of Nuclear Medicine and Molecular Imaging, Inc.
}

Key Words: pulmonary embolism; ventilation perfusion scintigraphy; single photon emission computed tomography; practices

J Nucl Med 2015; 56:1212-1217

DOI: 10.2967/jnumed.115.157743

$\mathbf{I}$ has been more than 50 y since lung scintigraphy was first described for the diagnosis of pulmonary embolism (PE). Since that time, the examination has evolved greatly with respect to equipment, radiopharmaceuticals, and imaging algorithms, along with perception among nuclear medicine and referring physicians.

Although performed $25 \mathrm{y}$ ago, using imaging equipment and ventilation agents that would currently be considered obsolete in most centers, the Prospective Investigation of Pulmonary Embolism Diagnosis study (PIOPED) study remains the landmark accuracy study in the eyes of many clinicians (1). The diagnostic performance of planar ventilation/perfusion (V/Q) scanning was then insufficient to allow a binary reporting approach (PE or no PE). Therefore, probabilistic reporting criteria were proposed, resulting in a high proportion of nondiagnostic tests. Subsequently, these criteria were refined and other diagnostic criteria introduced to simplify the interpretation (2-5). To date, all major management outcome studies assessing diagnostic strategies based on V/Q scintigraphy have been performed using planar scintigraphy interpreted with the probabilistic reporting criteria or its derivatives (6-9). Although complex to apply, these strategies were shown to safely rule out PE in large management outcome studies that demonstrated excellent patient outcomes when the diagnostic and therapeutic decisions were made on the basis of planar imaging. These studies made clinicians confident that planar V/Q scanning is an "established test for diagnosing PE" (10). Furthermore, scintigraphic strategies may be more appropriate than CT pulmonary angiography (CTPA)-based strategies with regards to the current concern about overdiagnosis and overtreatment of PE (8). However, use of probabilistic terminology and a relatively high rate of indeterminate results have led to a decreasing popularity of V/Q scans as compared with CTPA with its binary and easily understandable, if not necessarily more accurate, reporting approach.

In recent years, the technology around V/Q scintigraphy has rapidly evolved. Development of new imaging equipment and newer and more optimal radiopharmaceuticals has led to the 
introduction of SPECT V/Q scintigraphy, which has been reported to improve the diagnostic performances of the test (11-14) and significantly decrease the proportion of nondiagnostic studies (1517). The guidelines of the European Association of Nuclear Medicine (EANM) for V/Q scintigraphy strongly recommend use of SPECT and advocate the use of binary reporting terminology in most cases (18). The Society of Nuclear Medicine and Molecular Imaging is less definitive but also allows the use of V/Q SPECT (19). In a systematic review of the literature published by a group of experts in 2009, the promising results of V/Q SPECT were noted, but the group recommended that a large prospective evaluation was needed (20). Although V/Q SPECT has many proponents within the nuclear medicine community, publications from nonnuclear medicine specialists often still focus on planar imaging, in particular referring to its high rate of nondiagnostic examinations $(21,22)$. Some have described V/Q SPECT as a new technology requiring definitive validation $(10,23)$. Indeed, a large management outcome study based on the V/Q SPECT for PE diagnosis is lacking. More recently, the introduction of hybrid SPECT/CT systems has enabled simultaneous acquisition of V/Q SPECT and CT scanning of the lungs. A low-dose CT, in addition to V/Q imaging (24), or in lieu of ventilation images, has also been proposed for diagnosing PE (25).

There are currently no data available regarding current practices in nuclear medicine centers regarding the diagnosis of acute PE. In particular, little is known concerning the proportion of centers using SPECT or SPECT/CT rather than planar imaging, nor are there data regarding which criteria are currently used to interpret

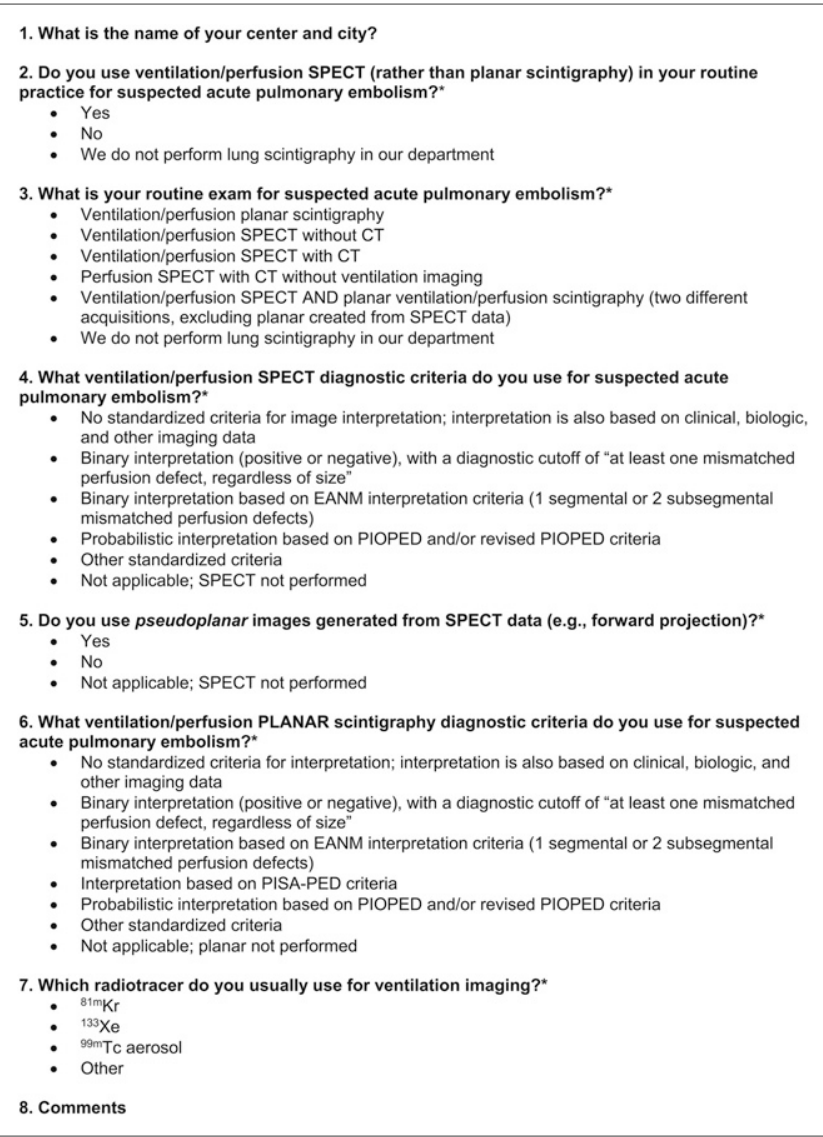

FIGURE 1. Common questionnaire. * Single-best-answer multiple-choice question. planar and V/Q SPECT. The aim of this study was, therefore, to assess these practices in nuclear medicine centers.

\section{MATERIALS AND METHODS}

\section{Questionnaire}

A survey was developed using an Internet-based survey tool (www. surveymonkey.com). Length and complexity were limited to improve compliance and the response rate. The questionnaire encompassed image acquisition, diagnostic criteria for SPECT and planar interpretation, and use of pseudoplanar images (planarlike images derived from SPECT data) and radiotracer for ventilation images (Fig. 1). The survey comprised single-best-answer multiple-choice questions. The software made it mandatory to answer one question before accessing the following one. All questions included a Non Applicable proposal to avoid random default response.

\section{Collection of Data}

The survey was initially conducted in France in April and May 2014 with the objective of obtaining 1 response per institution. Two hundred ten institutions were listed on the basis of the directory from the French Society of Nuclear Medicine (SFMN) (www.sfmn.org). First, an e-mail including an Internet link to the survey was sent to 1 of the physicians from each center, typically the head of the department. A few days later, a reminder e-mail was sent to the departments that had not responded. Finally, departments were solicited by telephone: 1 nuclear medicine physician was contacted and asked to respond to the questionnaire.

To obtain more generalizable data from different continents, the survey was then extended to Canada from September to November 2014 and in Australia from October to December 2014. In collaboration with the Canadian Association of Nuclear Medicine (CANM) and the Australasian Association of Nuclear Medicine Specialists (AANMS), the survey was circulated to their memberships via 2 sets of e-mails. Australian departments were also subsequently solicited by telephone. Concordant with the survey design initially performed in France, 1 response per center was consolidated in Australia and Canada. In Canada, 158 nuclear medicine institutions were listed on the basis of the directory from the CANM, and the number of institutions in Australia was estimated to be approximately 170 . In the few cases of discordant results between physicians from the same institution, the most frequent response was chosen. If there were only 2 discordant responses from the same department, the first complete response was recorded.

\section{RESULTS}

\section{Data Collection}

In France, 209 responses were collected (response rate, 209/210 = 99.5\%): $78(37 \%)$ after the first e-mail, $57(27 \%)$ after the second e-mail, and 74 (35\%) after a phone call. In Canada, 75 responses were collected covering 48 sites (response rate, $48 / 158=30 \%$ of centers) (respectively, 36 [75\%] and 12 [25\%] after the first and reminder e-mails). In Australia, 85 responses were collected covering 74 sites (response rate, $74 / 170=44 \%$ ) (respectively, 35 [47\%] and 20 [27\%] after the first and reminder e-mails and 19 [26\%] by phone call). Overall, 331 different institutions were assessed among the 3 countries: 74 (22\%) in Australia, 48 (14\%) in Canada, and 209 (63\%) in France.

\section{Routine Examination for Suspicion of Acute PE}

Of the 331 centers, 12 did not perform lung scintigraphy. Among the 319 remaining departments, $231(72 \%)$ routinely used V/Q SPECT in the context of acute PE suspicion (Australia, 85\%; Canada, 77\%; France, 67\%).

Overall, the routine examination in the setting of suspicion of acute PE was a V/Q SPECT without CT in 116 (36\%), a V/Q 


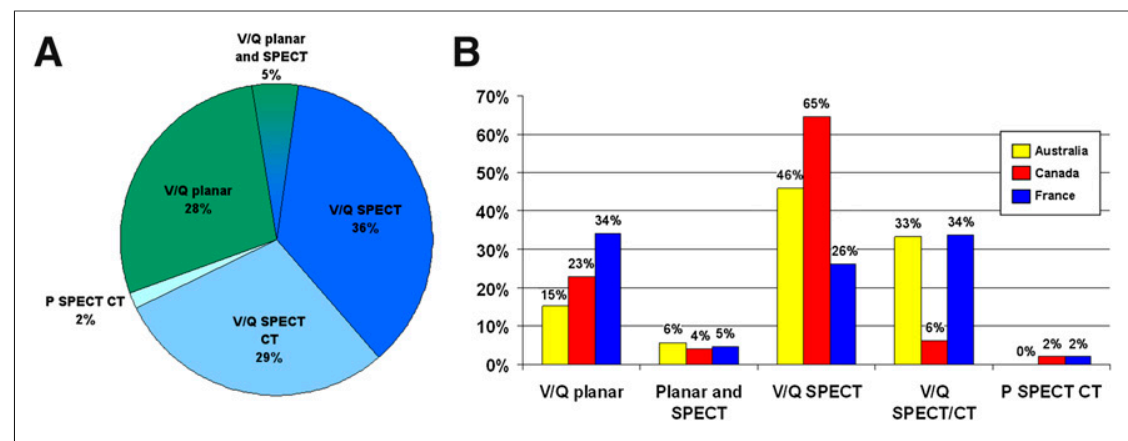

FIGURE 2. What is your routine examination for suspicion of acute PE? (A) Overall results from the 3 countries. (B) Results in Australia, Canada, and France. the clinicians (without usefulness for interpretation) in 19 (38\%) institutions.

\section{V/Q PLANAR Interpretation Criteria}

Of the 311 centers assessed, 99 indicated that they did not perform V/Q planar imaging. Among the 232 remaining institutions, EANM criteria were used in $35 \%$, the PIOPED criteria in $29 \%$, no standardized criteria in $21 \%$, a binary approach with a diagnostic cutoff of 1 subsegmental mismatched defect in $20 \%$, and the Prospective Investigative Study of Acute Pulmonary Embolism Diagnosis criteria in 1\% (Fig. 4).
SPECT CT in 94 (29\%), a planar V/Q scan in 90 (28\%), both planar and SPECT acquisitions in $15(5 \%)$, and a perfusion SPECT CT (without ventilation images) in $5(2 \%)$ departments (Fig. 2A). There was a marked variability between the 3 countries in the proportions of centers using V/Q planar, V/Q SPECT, and V/Q SPECT CT, respectively, 15\%, 46\%, and 33\% in Australia; $23 \%, 65 \%$, and $6 \%$ in Canada; and 34\%, 26\% , and 36\% in France (Fig. 2B).

Among the institutions performing V/Q SPECT, a CT was associated in $46 \%$ of cases (Australia, 42\%; Canada, 11\%; France, $58 \%)$.

\section{V/Q SPECT Interpretation Criteria}

Sixty-one centers responded that they did not perform V/Q SPECT. Of the 270 remaining centers, the most commonly used interpretation criteria were those of the EANM (binary interpretation with a diagnostic threshold of 1 segmental or 2 subsegmental mismatched defects), which were used in $60 \%$ of cases; $20 \%$ did not use standardized criteria, $11 \%$ used a binary reporting interpretation with a diagnostic cutoff of 1 subsegmental mismatched defect, and $8 \%$ used the revised PIOPED criteria. The proportion of sites using the various V/Q SPECT interpretation criteria was broadly consistent among the 3 countries (Fig. 3).

\section{Use of Pseudoplanar Images}

Overall, $63 \%$ of institutions used planar images generated from SPECT data, so-called pseudoplanar images (82\% in Australia, $63 \%$ in Canada, 56\% in France). In Australia, a more detailed question was provided to assess the role of these additional images. Among the 53 departments producing pseudoplanar images, the aim was for primary interpretation in $3(8 \%)$, to confirm/correlate SPECT interpretation in $31(58 \%)$, and to provide planar images to

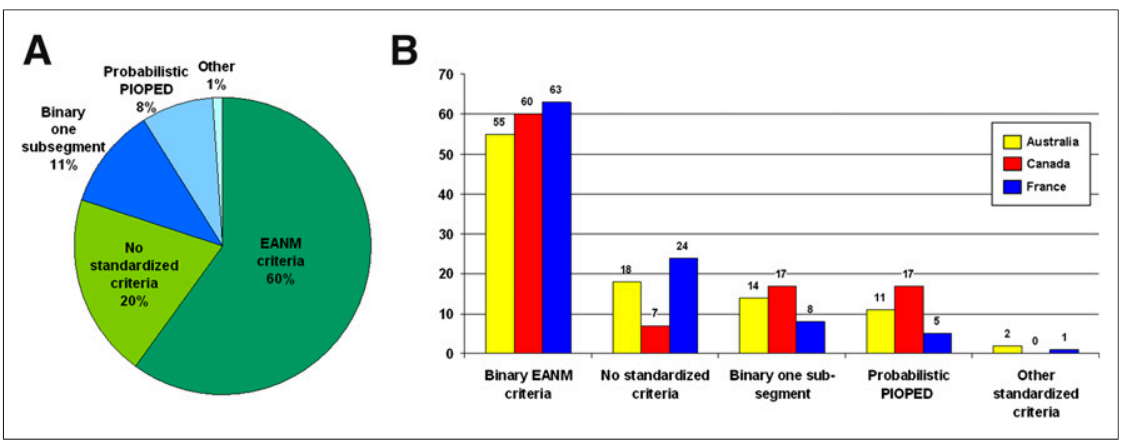

FIGURE 3. What ventilation/perfusion SPECT diagnostic criteria do you use for suspicion of acute PE? (A) Overall results from the 3 countries. (B) Results in Australia, Canada, and France.

\section{Radiotracer for Ventilation Imaging}

The ventilation agent used for V/Q imaging varied among the 3 countries assessed. In France, $83 \%$ of institutions used a radiolabeled aerosol ( ${ }^{99 \mathrm{~m}} \mathrm{Tc}$-diethylene triamine pentaacetic acid (DTPA) or ${ }^{99 \mathrm{~m}}$ Tc-labeled technegas), with the remaining $17 \%$ using ${ }^{81 \mathrm{~m}} \mathrm{Kr}$. In Canada, 96\% used a radiolabeled aerosol ( ${ }^{99 m}$ Tc-DTPA, $17 \%$; ${ }^{99 m}$ Tc-labeled technegas, $79 \%$ ), and $4 \%$ used ${ }^{133} \mathrm{Xe}$. In Australia, only ${ }^{99 \mathrm{~m}} \mathrm{Tc}$-labeled technegas was used.

\section{DISCUSSION}

Several conclusions can be reached based on responses from 331 nuclear medicine departments in 3 different countries regarding V/Q scintigraphy for the diagnosis of acute PE. First, in these countries, SPECT has largely replaced planar imaging, with almost half of the SPECT studies also incorporating a low-dose CT. Second, criteria used for interpretation are inconsistent, especially for V/Q planar imaging.

The observation that a large majority of nuclear medicine centers (72\%) have switched from the traditional planar imaging to SPECT suggests that the nuclear medicine community considers V/Q SPECT to be a more appropriate test with higher diagnostic accuracy than planar scanning for diagnosing PE. By using 3dimensional imaging, V/Q SPECT has an undoubted technical advantage over conventional planar imaging through its ability to eliminate overlap of perfusion defects; its visualization of the medial-basal segment; and its ability to better assess the size, shape, and location of defects (26). Many studies have demonstrated the higher diagnostic performances of V/Q SPECT when compared with planar imaging for the diagnosis of PE (11-14). In particular, V/Q SPECT has been reported to dramatically reduce the proportion of nondiagnostic tests $(15,16,27)$. It is for these reasons that the majority of nuclear medicine physicians have adopted V/Q SPECT as routine for PE diagnosis.

However, $33 \%$ of institutions still perform planar V/Q scintigraphy, with $5 \%$ of departments acquiring both planar and SPECT images. The reason why these institutions have not adopted V/Q SPECT is unclear and was not specifically elucidated in the study. Possible barriers preventing the transition from planar to SPECT imaging include resistance to change, greater familiarity and experience with planar imaging, concerns relating to increased image acquisition times, and the lack of a suitable ventilation agent 


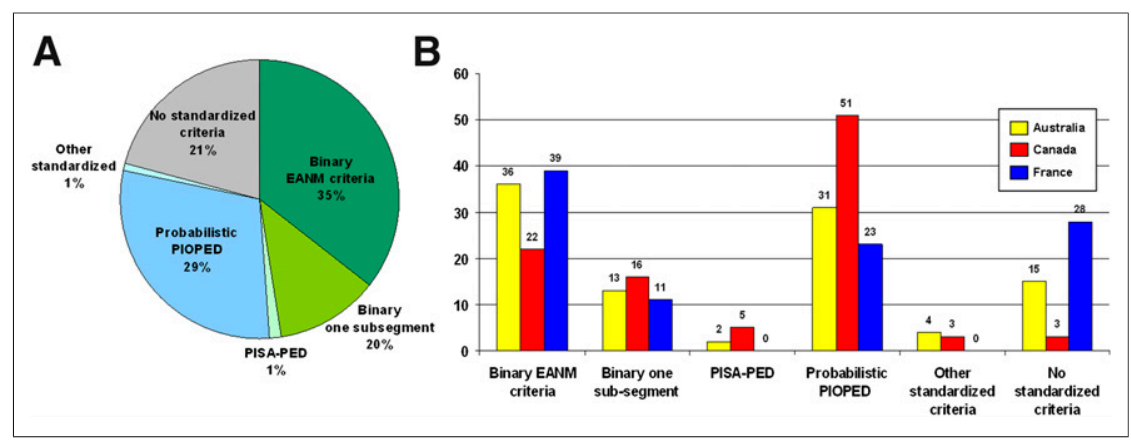

FIGURE 4. What ventilation/perfusion PLANAR scintigraphy diagnostic criteria do you use for suspicion of acute PE? (A) Overall results from the 3 countries. (B) Results in Australia, Canada, and France.

(26). Some physicians may consider that there is no need to change because planar V/Q is a validated and well-established examination. Other physicians, especially those performing both SPECT and planar acquisition, reported a willingness to switch from planar to SPECT V/Q but were constrained by a reluctance of the clinicians to adopt this new imaging method.

Although the proportion of nuclear medicine physicians performing SPECT acquisitions for diagnosis of PE is high in the countries surveyed, references to V/Q scanning in the medical literature by nonnuclear medicine specialists frequently refer to planar imaging, often with reference to its high nondiagnostic rates $(21,22)$. In the few publications that reference V/Q SPECT, it is often portrayed as a test with future potential applicability, rather than an examination in routine use today (10). Although current clinical guidelines make reference to planar V/Q imaging (10), and presumably many referring clinicians assume that this is how V/Q is still performed, our survey shows that in at least the 3 countries surveyed, SPECT has largely replaced planar imaging in the investigation of PE. This raises concern that there is a lack of communication between nuclear medicine physicians and referring physicians about how the test is performed. This discrepancy may be related to several factors. First, studies describing V/Q SPECT have been mostly published in nuclear medicine journals rather than in high-impact general internal medicine journals. In contrast, methods such as CTPA, V/Q planar, or D-dimer testing are more widely described in the nonimaging literature. Furthermore, V/Q SPECT has not achieved the same standard of validation as others diagnostic tests including planar V/Q imaging (1,6-9), CTPA (28-30), or D-dimer testing $(31,32)$, which may impede its widespread acceptance by clinicians. Indeed, although there is an extensive literature reporting the superior diagnostic performances of V/Q SPECT as compared with V/Q planar, the exact accuracy of V/Q SPECT for PE diagnosis is still unknown $(20,23)$. Most importantly, although all diagnostic tests and strategies (including CTPA, planar V/Q, or Ddimer) for PE diagnosis have been validated in large management outcome studies, such a study with V/Q SPECT is still lacking. It is likely that achieving the same standard of validation will be an essential step in convincing clinicians, and especially venous thromboembolism specialists, of the usefulness of V/Q SPECT for diagnosis of PE.

Among institutions using V/Q SPECT, 46\% simultaneously acquired CT images. The relatively high proportion of institutions that have adopted this approach would suggest that imaging specialists consider the CT to be useful for the interpretation of V/Q SPECT. However, there was a marked variability between the 3 countries. In
France, where the proportion of centers using V/Q planar was the highest, $58 \%$ of institutions performing SPECT also acquired CT images. The proportion was $42 \%$ in Australia and only $11 \%$ in Canada. These results cannot be fully explained by differences in the availability of hybrid cameras. Indeed, although most Canadian institutions do have a hybrid SPECT/CT system, only $11 \%$ of centers performed V/Q SPECT CT acquisition. Dosimetric considerations may be a contributing factor, although the additional radiation dose is only on the order of $1 \mathrm{mSv}$ whereas overall radiation dose of a CTPA is on the order of 8-20 mSv (11). Difference in cost and in reimbursement policies could also play a role in some jurisdictions. In addition, there are much less data validating the use of V/Q SPECT/CT as compared with V/Q SPECT alone. Studies have consistently reported a higher specificity of the test $(24,33)$, which may be of relevance in the setting of the increasing concern about a possible overtreatment of PE and a trend toward the use of extended duration of anticoagulation therapy (34). An additional low-dose CT also allows the detection of alternative diagnoses, which has been reported as an advantage of CTPA (35). A limited number of institutions routinely use a low-dose CT in lieu of ventilation imaging (Q SPECT/CT). Recent data demonstrated similar sensitivity but lower specificity, with a high risk of false-positive results when compared with V/Q SPECT (24).

The second part of the survey was dedicated to the interpretation criteria. There is an extensive literature on the topic, with multiple criteria proposed for interpretation, likely as a result of the probabilistic reporting approach proposed in the PIOPED study. For V/Q SPECT, the most commonly used criteria $(60 \%)$ were those proposed by the EANM, that is, a binary approach (PE or no PE) with a diagnostic cutoff of "1 segmental or 2 subsegmental perfusion mismatched defects" (18). This diagnostic cutoff has been reported to be optimal for PE diagnosis (36), and a recent observational study confirmed the diagnostic exclusion safety of a diagnostic management including a V/Q SPECT study interpreted with these criteria (37). Only $8 \%$ of institutions used the probabilistic approach for interpretation of SPECT, compared with $29 \%$ for planar. Our survey demonstrated that the shift from planar to SPECT imaging has also been associated with a replacement of probabilistic reporting with a more binary reporting approach.

Although there is some variability in the reporting criteria used for SPECT imaging, there is much greater inconsistency in the interpretation of planar imaging. In the survey, $29 \%$ of institutions use the probabilistic reporting approach, allowing the integration the V/Q scan results into validated diagnostic algorithms. However, most centers performing planar imaging use a binary reporting approach, with $35 \%$ of respondents using the EANM diagnostic cutoff and $20 \%$ of institutions using a 1 subsegmental cutoff. This is a relevant finding for referring specialists, as the reporting criteria used may not be those that have been validated in large outcome management studies $(6-9,38)$. Overall, only $10 \%$ of centers currently use planar V/Q interpreted according to the probabilistic criteria, highlighting our observation that the approach to image acquisition and the reporting criteria used for V/Q scanning have changed in recent years, which may not be understood by referring clinicians.

Planarlike images can be derived from SPECT data using several approaches. In our survey, 63\% of institutions performing V/Q SPECT also generate pseudoplanar images. In Australia, departments 
were further asked about the rationale of producing such images. For $36 \%$ of institutions, the aim is just to provide planar images to the referring clinicians, with the pseudoplanar images not being used for interpretation. Fifty-eight percent of institutions use them to confirm or correlate SPECT image interpretation. Although summed planar images have been shown to approximate true planar images, they are not perfectly concordant and may blur out small perfusion defects $(39,40)$. Although the concordance between reprojected and true planar images may be better (39), how to manage discrepancies between SPECT and pseudoplanar images, and how they should be used by both reporting specialists and referring specialists, remains unresolved.

This study has some limitations. First, the questionnaire was composed of a small number of multiple-choice questions, which limited the possible responses. However, all questions included a response of other, which was chosen in a limited number of cases, suggesting that most physicians found an adequate response among the propositions. Second, only 1 answer per institution was consolidated to conform to the initial survey design performed in France. Accordingly, we did not assess the variability of practices among physicians from a same institution, which would be a subject of interest. Third, the participation rate was $30 \%$ in Canada and $44 \%$ in Australia, which could bias the results because nonresponders may hold divergent practices. Nonetheless, the participation rate was almost universal (209/210 departments) in France, where the survey demonstrated the same trend in the use of SPECT and similar inconsistency with regard to the interpretation criteria. Fourth, the conclusions drawn relate to the 3 countries that were surveyed and it may not be possible to extrapolate the results more broadly to other countries. Access to hybrid scanners varies around the world, and in particular, there is differing access to various scintigraphic ventilation agents. Of particular note is that technegas or Krypton are not available in the United States, which may be a contributing factor to the low adoption of V/Q SPECT imaging in that country, although some suggest that ${ }^{99 \mathrm{~m} T c-D T P A}$ and ${ }^{133} \mathrm{Xe}$ may be used.

\section{CONCLUSION}

This survey of current practices in nuclear medicine departments, spanning 3 countries, demonstrated that SPECT has largely replaced planar imaging for diagnosing $\mathrm{PE}$ and that interpretation criteria are variable. These trends may not be understood by referring clinicians, which highlights the need for further standardization of practices and better communication between nuclear medicine physicians and clinicians. Although validation studies have been done using V/Q planar, CTPA, and D-dimer, they are lacking with regard to the use of V/Q SPECT in the diagnostic pathway for suspected PE. Clinical trials validating the performance of SPECT would be of value to achieve uniform practices within nuclear medicine departments and to convince clinicians of the usefulness of V/Q SPECT for diagnosis of PE.

\section{DISCLOSURE}

The costs of publication of this article were defrayed in part by the payment of page charges. Therefore, and solely to indicate this fact, this article is hereby marked "advertisement" in accordance with 18 USC section 1734 . No potential conflict of interest relevant to this article was reported.

\section{ACKNOWLEDGMENTS}

We acknowledge the AANMS, the CANM, and the SFMN for their collaboration. In particular, we thank Janine Sargeant and
Ann McGregor from the AANMS, Hélène Samson and Dr. JeanLuc Urbain from the CANM, and Dr. Gérald Bonardel from the SFMN for their assistance with the administration of the survey. We also thank the physicians who answered the questionnaire.

\section{REFERENCES}

1. PIOPED Investigators. Value of the ventilation/perfusion scan in acute pulmonary embolism: results of the prospective investigation of pulmonary embolism diagnosis (PIOPED). JAMA. 1990;263:2753-2759.

2. Gottschalk A, Sostman HD, Coleman RE, et al. Ventilation-perfusion scintigraphy in the PIOPED study: part II-evaluation of the scintigraphic criteria and interpretations. J Nucl Med. 1993;34:1119-1126.

3. Sostman HD, Coleman RE, DeLong DM, Newman GE, Paine S. Evaluation of revised criteria for ventilation-perfusion scintigraphy in patients with suspected pulmonary embolism. Radiology. 1994;193:103-107.

4. Miniati M, Pistolesi M, Marini C, et al. Value of perfusion lung scan in the diagnosis of pulmonary embolism: results of the Prospective Investigative Study of Acute Pulmonary Embolism Diagnosis (PISA-PED). Am J Respir Crit Care Med. 1996;154:1387-1393.

5. Glaser JE, Chamarthy M, Haramati LB, Esses D, Freeman LM. Successful and safe implementation of a trinary interpretation and reporting strategy for V/Q lung scintigraphy. J Nucl Med. 2011;52:1508-1512.

6. Perrier A, Desmarais S, Miron MJ, et al. Non-invasive diagnosis of venous thromboembolism in outpatients. Lancet. 1999;353:190-195.

7. Wells PS, Ginsberg JS, Anderson DR, et al. Use of a clinical model for safe management of patients with suspected pulmonary embolism. Ann Intern Med. 1998;129:997-1005.

8. Anderson DR, Kahn SR, Rodger MA, et al. Computed tomographic pulmonary angiography vs ventilation-perfusion lung scanning in patients with suspected pulmonary embolism: a randomized controlled trial. JAMA. 2007;298:27432753.

9. Salaun PY, Couturaud F, Le Duc-Pennec A, et al. Noninvasive diagnosis of pulmonary embolism. Chest. 2011;139:1294-1298.

10. Konstantinides SV, Torbicki A, Agnelli G, et al. 2014 ESC guidelines on the diagnosis and management of acute pulmonary embolism. Eur Heart J. 2014;35:3033-3069, 3069a-3069k.

11. Roach PJ, Schembri GP, Bailey DL. V/Q scanning using SPECT and SPECT/CT. J Nucl Med. 2013;54:1588-1596.

12. Mortensen J, Gutte H. SPECT/CT and pulmonary embolism. Eur J Nucl Med Mol Imaging. 2014;41(suppl 1):S81-S90.

13. Le Duc-Pennec A, Le Roux PY, Cornily JC, et al. Diagnostic accuracy of singlephoton emission tomography ventilation/perfusion lung scan in the diagnosis of pulmonary embolism. Chest. 2012;141:381-387.

14. Bajc M, Olsson CG, Olsson B, Palmer J, Jonson B. Diagnostic evaluation of planar and tomographic ventilation/perfusion lung images in patients with suspected pulmonary emboli. Clin Physiol Funct Imaging. 2004;24:249-256.

15. Miles S, Rogers KM, Thomas $\mathrm{P}$, et al. A comparison of single-photon emission CT lung scintigraphy and CT pulmonary angiography for the diagnosis of pulmonary embolism. Chest. 2009;136:1546-1553.

16. Bajc M, Olsson B, Palmer J, Jonson B. Ventilation/perfusion SPECT for diagnostics of pulmonary embolism in clinical practice. J Intern Med. 2008;264:379387.

17. Leblanc M, Paul N. V/Q SPECT and computed tomographic pulmonary angiography. Semin Nucl Med. 2010;40:426-441.

18. Bajc M, Neilly JB, Miniati M, Schuemichen C, Meignan M, Jonson B. EANM guidelines for ventilation/perfusion scintigraphy: part 1-pulmonary imaging with ventilation/perfusion single photon emission tomography. Eur J Nucl Med Mol Imaging. 2009;36:1356-1370.

19. Parker JA, Coleman RE, Grady E, et al. SNM practice guideline for lung scintigraphy 4.0. J Nucl Med Technol. 2012;40:57-65.

20. Stein PD, Freeman LM, Sostman HD, et al. SPECT in acute pulmonary embolism. J Nucl Med. 2009;50:1999-2007.

21. Lapner ST, Kearon C. Diagnosis and management of pulmonary embolism. BMJ. 2013;346:f757.

22. Agnelli G, Becattini C. Acute pulmonary embolism. $N$ Engl J Med. 2010;363: 266-274.

23. Stein PD, Sostman HD, Matta F. Critical review of SPECT imaging in pulmonary embolism. Clin Transl Imaging. 2014;2:379-390.

24. Gutte H, Mortensen J, Jensen CV, et al. Detection of pulmonary embolism with combined ventilation-perfusion SPECT and low-dose CT: head-to-head comparison with multidetector CT angiography. J Nucl Med. 2009;50:19871992. 
25. Lu Y, Lorenzoni A, Fox JJ, et al. Non-contrast perfusion SPECT/CT: a new test for the expedited, high accuracy diagnosis of acute pulmonary embolism. Chest. January 2, 2014 [Epub ahead of print].

26. Roach PJ, Bailey DL, Schembri GP, Thomas PA. Transition from planar to SPECT V/Q scintigraphy: rationale, practicalities, and challenges. Semin Nucl Med. 2010;40:397-407.

27. Leblanc M, Leveillee F, Turcotte E. Prospective evaluation of the negative predictive value of V/Q SPECT using ${ }^{99 m}$ Tc-technegas. Nucl Med Commun. 2007;28: 667-672.

28. Stein PD, Fowler SE, Goodman LR, et al. Multidetector computed tomography for acute pulmonary embolism. N Engl J Med. 2006;354:2317-2327.

29. Righini M, Le Gal G, Aujesky D, et al. Diagnosis of pulmonary embolism by multidetector CT alone or combined with venous ultrasonography of the leg: a randomised non-inferiority trial. Lancet. 2008;371:1343-1352.

30. van Belle A, Buller HR, Huisman MV, et al. Effectiveness of managing suspected pulmonary embolism using an algorithm combining clinical probability, D-dimer testing, and computed tomography. JAMA. 2006;295:172-179.

31. Wells PS, Anderson DR, Rodger M, et al. Excluding pulmonary embolism at the bedside without diagnostic imaging: management of patients with suspected pulmonary embolism presenting to the emergency department by using a simple clinical model and d-dimer. Ann Intern Med. 2001;135:98-107.

32. Righini M, Van Es J, Den Exter PL, et al. Age-adjusted D-dimer cutoff levels to rule out pulmonary embolism: the ADJUST-PE study. JAMA. 2014;311:1117-1124.
33. Roach PJ, Gradinscak DJ, Schembri GP, Bailey EA, Willowson KP, Bailey DL. SPECT/CT in V/Q scanning. Semin Nucl Med. 2010;40:455-466.

34. Kearon C, Akl EA, Comerota AJ, et al. Antithrombotic therapy for VTE disease: antithrombotic therapy and prevention of thrombosis, 9th ed-American College of Chest Physicians evidence-based clinical practice guidelines. Chest. 2012;141: e419S-494S.

35. Ling IT, Naqvi HA, Siew TK, Loh NK, Ryan GF. SPECT ventilation perfusion scanning with the addition of low-dose CT for the investigation of suspected pulmonary embolism. Intern Med J. 2012;42:1257-1261.

36. Le Roux PY, Robin P, Delluc A, et al. V/Q SPECT interpretation for pulmonary embolism diagnosis: which criteria to use? J Nucl Med. 2013;54:1077-1081.

37. Le Roux PY, Palard X, Robin P, et al. Safety of ventilation/perfusion single photon emission computed tomography for pulmonary embolism diagnosis. Eur J Nucl Med Mol Imaging. 2014;41:1957-1964.

38. Sostman HD, Miniati M, Gottschalk A, Matta F, Stein PD, Pistolesi M. Sensitivity and specificity of perfusion scintigraphy combined with chest radiography for acute pulmonary embolism in PIOPED II. J Nucl Med. 2008;49:1741-1748.

39. Harris B, Bailey DL, Roach PJ, et al. A clinical comparison between traditional planar V/Q images and planar images generated from SPECT V/Q scintigraphy. Nucl Med Commun. 2008;29:323-330.

40. Le Roux PY, Abgral R, Jaffrelot M, et al. Diagnosis of pulmonary embolism: planar images generated from V/Q SPECT are not a reliable substitute for traditional planar V/Q scan. Nucl Med Commun. 2012;33:695-700. 